Alterstice
Revue internationale de la recherche interculturelle
International Journal of Intercultural Research
Revista International de la Investigacion Intercultural

\title{
Exploration de l'expérience de la maternité chez des jeunes femmes haïtiennes issues du milieu rural : enjeux économiques, culturels et affectifs
}

\section{Gabrièle Gilbert et Sophie Gilbert}

Volume 7, numéro 2, 2017

URI : https://id.erudit.org/iderudit/1052572ar

DOI : https://doi.org/10.7202/1052572ar

Aller au sommaire du numéro

Éditeur(s)

Alterstice

ISSN

1923-919X (numérique)

Découvrir la revue

Citer cet article

Gilbert, G. \& Gilbert, S. (2017). Exploration de l'expérience de la maternité chez des jeunes femmes haïtiennes issues du milieu rural : enjeux économiques, culturels et affectifs. Alterstice, 7(2), 91-104. https://doi.org/10.7202/1052572ar

\section{Résumé de l'article}

Notre étude constitue un volet, ciblant les nouvelles mères, d'un projet qui vise à implanter un réseau de services communautaires en santé mentale dans la ville de Grand-Gôave en Haïti. Ces services sont dispensés par des aidants naturels sur place, dont le travail est supervisé à distance par des professionnels en santé mentale montréalais. Ce type de partenariat maximise les différences culturelles, ce qui se traduit par des divergences entre les perceptions de la problématique et de l'intervention à offrir, ainsi que par une complexification de l'implantation et de l'évaluation des services. Selon un modèle de recherche-action qualitative et par le biais de deux études de cas, de deux groupes de discussion ainsi que d'observations menées sur place, notre étude visait à mieux comprendre ce qui caractérise la trajectoire, la problématique ainsi que les besoins des utilisatrices et à voir ce que les services peuvent leur apporter, en particulier en matière de prévention de la violence familiale. D’après notre analyse thématique, ces nouvelles mères seraient exposées à de nombreux facteurs de risque socioculturels de grossesses accidentelles, en particulier le patriarcat, des tabous culturels et spirituels, une éducation sexuelle limitée ainsi qu’un accès réduit à l'avortement. De plus, leur expérience de la maternité serait caractérisée par l'absence de soutien ainsi que par des sentiments négatifs et un lien mère-enfant ambivalent, qui constituent des facteurs de risque de violence parentale. Nous proposons d'ajuster les interventions afin de soutenir le vécu maternel de ces jeunes femmes, en agissant tant au niveau individuel que social, et en soutenant les motivations spirituelles et culturelles à la maternité déjà présentes chez celles-ci afin de les utiliser comme levier à la résilience. 


\section{7}

ARTICLE HORS THÈME

\section{Exploration de l'expérience de la maternité chez des jeunes femmes haïtiennes issues du milieu rural : enjeux économiques, culturels et affectifs}

Gabrièle Gilbert ${ }^{1}$ et Sophie Gilbert ${ }^{1}$

\section{Résumé}

Notre étude constitue un volet, ciblant les nouvelles mères, d'un projet qui vise à implanter un réseau de services communautaires en santé mentale dans la ville de Grand-Gôave en Haïti. Ces services sont dispensés par des aidants naturels sur place, dont le travail est supervisé à distance par des professionnels en santé mentale montréalais. Ce type de partenariat maximise les différences culturelles, ce qui se traduit par des divergences entre les perceptions de la problématique et de l'intervention à offrir, ainsi que par une complexification de l'implantation et de l'évaluation des services. Selon un modèle de recherche-action qualitative et par le biais de deux études de cas, de deux groupes de discussion ainsi que d'observations menées sur place, notre étude visait à mieux comprendre ce qui caractérise la trajectoire, la problématique ainsi que les besoins des utilisatrices et à voir ce que les services peuvent leur apporter, en particulier en matière de prévention de la violence familiale. D’après notre analyse thématique, ces nouvelles mères seraient exposées à de nombreux facteurs de risque socioculturels de grossesses accidentelles, en particulier le patriarcat, des tabous culturels et spirituels, une éducation sexuelle limitée ainsi qu'un accès réduit à l'avortement. De plus, leur expérience de la maternité serait caractérisée par l'absence de soutien ainsi que par des sentiments négatifs et un lien mère-enfant ambivalent, qui constituent des facteurs de risque de violence parentale. Nous proposons d'ajuster les interventions afin de soutenir le vécu maternel de ces jeunes femmes, en agissant tant au niveau individuel que social, et en soutenant les motivations spirituelles et culturelles à la maternité déjà présentes chez celles-ci afin de les utiliser comme levier à la résilience.

\section{Rattachement des auteures}

${ }^{1}$ Université du Québec à Montréal, Montréal, Canada

\section{Correspondance}

gilbert.gabriele@courrier.uqam.ca

\section{Mots clés}

maternité, monoparentalité, santé mentale, précarité, culture, famille, violence familiale, organisme communautaire, père absent, lien mère-enfant, partenariat transculturel

\section{Pour citer cet article}

Gilbert, G. et Gilbert, S. (2017). Exploration de l'expérience de la maternité chez des jeunes femmes haïtiennes issues du milieu rural : enjeux économiques, culturels et affectifs. Alterstice, 7(2), 91-104. 


\section{Introduction}

En Haïti, et plus particulièrement en milieu rural, les femmes se heurtent régulièrement à un manque d'accès à l'information concernant leurs droits sexuels et reproductifs, à une reconnaissance limitée de ces droits ainsi qu'à une incidence élevée de la violence et de la manipulation sexuelle, conjugale et économique (Ministère à la Condition féminine et aux Droits des Femmes [MCFDF], 2006).

La faible d'utilisation de contraceptifs qui en résulte amène plusieurs femmes à tomber enceintes à un âge précoce (Chandra-Mouli, Camacho et Michaud, 2013) et à vivre des grossesses non planifiées. De plus, l'avortement est interdit en Haïti et, lorsqu'il est pratiqué clandestinement, il comporte des risques pour la santé de la mère (Logeart, 2014). Ainsi, plusieurs de ces femmes choisissent de mener à terme leurs grossesses, certaines de ces dernières étant non désirées (Maternowska, 2006).

N'ayant pas terminé leurs études et étant confrontées au taux élevé de chômage caractéristique des communautés rurales en Haïti (Paul, Dameus et Garrabe, 2011), elles dépendent financièrement de leur famille et risquent de subir du rejet de la part de cette dernière (Laroche, 2012). De plus, l'absence du père est particulièrement prégnante : $47 \%$ des familles haïtiennes étaient monoparentales en 2011 (Joseph et Kahou), les enfants étant généralement sous la garde de la mère. Dans le cas où le père est présent, il n'est pas rare que ce dernier fasse preuve d'un désinvestissement sur le plan psychique par rapport à la famille (Flambert-Chéry, 2013).

Les sentiments négatifs issus de ce manque de soutien, de ces rejets et de ces pertes, ajoutés à des conditions de vie précaires, exposent ces jeunes mères à un risque de fragilisation psychologique, susceptible de mener à la dépression. Ce problème de santé mentale risque d'affecter l'enfant et la relation avec celui-ci, se traduisant notamment par des situations de négligence ou de maltraitance (Organisation mondiale de la santé, 2014), qui ont un impact délétère sur le développement physique et psychosocial de l'enfant (Benarous, Guilé, Consoli et Cohen 2014).

Ces risques pour la mère et sa progéniture sont d'autant plus préoccupants qu'en Haïti, la rareté, la distance ainsi que le coût élevé associé aux soins médico-psychosociaux spécialisés rendent ces derniers difficilement accessibles à la majorité de la population (Richard, 2015). C'est dans ce contexte de pénurie de services qu'est né l'organisme à but non lucratif Groupe de Santé Mentale (GROSAME) de Grand-Goâve, partenaire de notre recherche (Grosame Grand-Goâve, 2016).

\section{GROSAME}

GROSAME a pour but, depuis 2006, d'offrir des services de première ligne en santé mentale à la population de la ville de Grand-Goâve en Haïti. Pour ce faire, des Haïtiens de formations diverses, auparavant impliqués dans cette communauté comme aidants naturels et ayant reçu une formation en relation d'aide, sont temporairement employés à titre de travailleurs communautaires. Les activités et les interventions menées par ces derniers ont été supervisées sur place par un psychologue haïtien et à distance (par Skype) par des professionnels de la santé mentale de Montréal (Gilbert, Benjamin, Da, Toussaint et Lecomte, 2015).

Outre les problèmes liés à la communication par Internet, les différences de perception (dues à des facteurs culturels) entre les superviseurs montréalais et les intervenants locaux en ce qui concerne la problématique et l'intervention à offrir se sont traduites par une complexification de l'implantation et de l'évaluation des services. Ces obstacles ont conduit à un questionnement sur l'adaptation des services aux problématiques complexes et aux besoins des utilisatrices du volet de GROSAME destiné aux nouvelles mères.

Afin de combler ces lacunes, nous avons eu comme objectifs de mieux comprendre 1) ce qui caractérise la trajectoire des nouvelles mères utilisatrices des services, 2) leur problématique actuelle et 3 ) leurs besoins, en particulier en ce qui concerne le risque de violence familiale. 


\section{Méthodologie}

Nos participantes devaient être des jeunes femmes haïtiennes primipares dont la grossesse n'était pas planifiée et qui ont reçu des services offerts par GROSAME. Neuf femmes correspondant à ces critères se sont portées volontaires pour participer à des groupes de discussion (I'un avec 4 participantes et l'autre avec 5 participantes) d'une durée d'une heure environ chacun. Ce dispositif a favorisé les interactions entre nos participantes, ce qui a permis de stimuler leur réflexion et de faire émerger chez elles différentes perceptions, attitudes et croyances en lien avec leur situation.

Deux de nos participantes ont également chacune pris part à deux entretiens individuels (d'environ une heure) afin $d^{\prime}$ 'approfondir certains thèmes abordés lors des groupes de discussion ( $p$. ex. leur histoire personnelle et familiale ou leur vécu par rapport à la grossesse). Ces dernières se sont également prêtées à des observations à domicile qui, avec leur cadre "naturel» (jeu spontané mère-enfant et échanges informels), ont permis d'enrichir notre compréhension de la réalité quotidienne des nouvelles mères chez qui ces observations ont eu lieu.

Plus précisément, nous avons opté pour un devis qualitatif d'approche participative. Notre démarche a permis de faire émerger, de façon inductive (Paillé et Mucchielli, 2012), certains éléments saillants de la problématique des nouvelles mères. Les modalités de recueil de données ont consisté en des entretiens semi-directifs menés en créole et dont la question d'entame était volontairement ouverte, afin de suivre le fil conducteur du discours des participantes (Gilbert, 2009). Cette façon de faire nous a donné accès à la complexité de l'expérience des mères, de manière à ce que le "savoir " recherché puis analysé soit le leur plutôt que le nôtre ou celui des intervenants locaux.

En outre, notre appropriation préalable des données recueillies par GROSAME concernant les jeunes mères bénéficiant des services de l'organisme a permis de dégager des éléments de questionnements qui ont été inclus dans nos canevas d'entretiens. Nous avons ainsi exploré plusieurs thématiques en lien avec la trajectoire, la problématique et les besoins de nos participantes (voir annexe).

La réduction des données (provenant des transcriptions et des comptes-rendus d'observations) a été effectuée par une analyse descriptive thématique (Paillé et Mucchielli, 2012) afin que nos résultats demeurent au plus près de la réalité et du discours des participantes. De plus, l'atteinte d'un consensus entre les deux chercheures (les auteures) dans la thématisation ainsi que l'attention portée aux réactions subjectives (de même qu'aux biais socioculturels) ont favorisé la rigueur de notre analyse.

\section{Résultats}

L'âge moyen de nos participantes était de 25 ans, celui de leur enfant de 2 ans. Le tableau 1 permet de dresser un portrait détaillé de certaines de leurs caractéristiques sociodémographiques. Quant à l'expérience de la maternité de nos participantes, elle peut être illustrée par la figure suivante (figure 1).

\section{Figure 1. Expérience de la maternité}

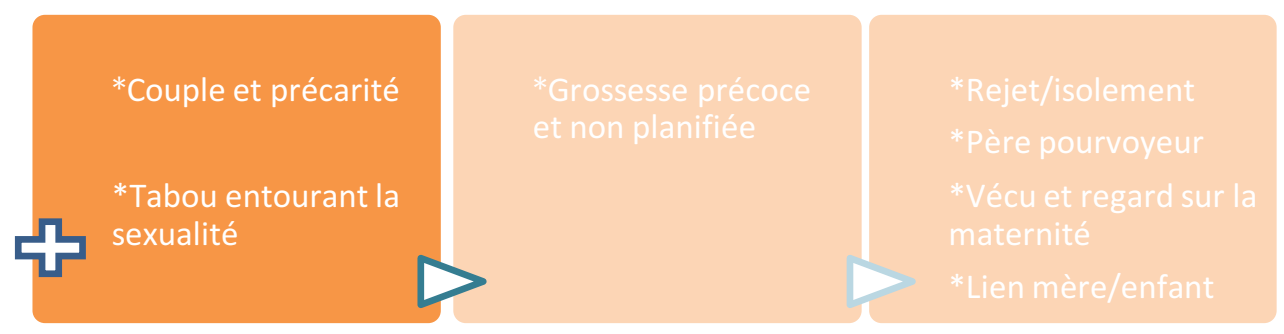




\section{Tableau 1. Caractéristiques des participantes}

$\begin{array}{llc}\text { Données sociodémographiques } & \text { Catégories } & 2 \\ \text { État matrimonial } & \text { Mariée } & 3 \\ & \text { Union libre } & 4 \\ \text { Logement } & \text { Célibataire } & 5 \\ & \text { Avec le père de l'enfant } & 2 \\ & \text { Seule } & 1 \\ \text { Situation parentale } & \text { Famille } & 1 \\ \text { Autres ressources fréquentées } & \text { Amis } & 4 \\ & \text { Monoparentalité } & 5 \\ \text { Sources de revenus } & \text { Biparentalité } & 6 \\ & \text { Église } & 2 \\ \text { Niveau de scolarité } & \text { Organisme à but non lucratif } & 3 \\ & \text { Emploi } & 4 \\ & \text { Le père de l'enfant } & 2\end{array}$

\section{Couple et précarité}

Les entretiens individuels ont permis de relever l'existence d'un lien entre la formation des couples et la dépendance financière des jeunes femmes. En effet, comme l'illustre Mirlyn, un conjoint peut représenter une solution à la précarité :

Ce qui est arrivé c'est que j'ai commencé à fréquenter un garçon. Comme ça, j'aurais pu continuer mes études. Mais malheureusement... je suis tombée enceinte. [Mwen te arivé tonbe nan renmen pou'm te ka kontinye étid mwen. Men malerezman m'vin tonbe ansent.]

De plus, le pouvoir étant culturellement attribué à l’homme en Haïti (Gage et Hutchinson, 2006), ces jeunes femmes qui, du fait de leur situation de grande précarité, dépendent financièrement de lui n'ont pas vraiment de pouvoir de négociation en ce qui concerne les relations sexuelles (Maternowska, 2006). Comme le souligne la littérature, nos résultats montrent qu'afin d'éviter de perdre un homme (et son soutien financier), ces femmes seraient susceptibles de répondre à la pression de leur partenaire relative aux pratiques sexuelles non protégées par la soumission ou la résignation :

Le jour du malheur... il est allé en chercher [des préservatifs] mais il n’en a pas trouvé. Je lui ai dit que j'allais tomber enceinte et il m’a dit : "comment ça?". Je lui ai dit, mais il ne m’a jamais crue (Mirlyn). [Jou malè a rive... li al cheche'l, li pa jwen li. Mwen te di'l ke m'tap tonbe ansent. Li di'm 'sak fè sa? 'Li pat janm kwè'm, men m'te di'l sa.]

\section{Le caractère tabou de la sexualité}

Les participantes, lors des entretiens individuels, nous ont également confié méconnaître leur histoire familiale, et en particulier les conditions entourant leur propre naissance. Ces trous dans leur histoire semblent comblés par l'impression de répéter malgré elles l'histoire maternelle - dans ce cas, une situation de précarité. En ce qui concerne Mirlyn, il semblerait que sa mère (qui peine à accepter sa grossesse) n'ait pas voulu lui avouer qu'elle l'avait elle-même eue approximativement au même âge, et donc possiblement dans les mêmes conditions :

Nous n'avions pas l'habitude de parler de ça... je lui disais : "j'ai l'impression que tu m'as eue vers l'âge de 22 ans... » Mais elle n'a jamais voulu me le dire... moi j'ai l'impression que c'est à l'âge de 22 ans qu'elle m'a eue. (Mirlyn). [Nou pat kon pale de sa. M'te kon di'l... jan m'wè... li sanble se a 22 ans ou te fè'm... Men li pat janm vle di'm. Kou mwen menm, li sanble a 22ans li te fè'm.] 
En outre, selon l'une de nos participantes aux groupes de discussion, la sexualité en Haïti constitue un sujet que l'on ne maîtrise pas et dont on ne discute pas :

Chercheure : Est-ce que vos parents s'étaient assis avec vous pour parler de sexualité?

- Non, non! II n'y a pas eu de ça! Nos parents sont analphabètes... comment est-ce qu'ils auraient pu faire... ils ne connaissent pas toutes ces choses... [Eske paran nou te chita avè'n pou pale de seksyalite? Non, non! Pat gen bagay sa yo non! Paran nou se paran ki analfabèt... Kote yo pral... pat gen bagay sa yo.]

Une répercussion importante du tabou et du manque d'éducation sexuelle en Haïti consiste en des croyances erronées, tant chez les femmes que chez les hommes, en matière de sexualité (Maynard-Tucker, 1996), donnant lieu à une perception négative des contraceptifs :

Je ne voulais pas le mettre. Parce qu'ils disent qu'il y a une huile dans le préservatif... qu'il y a une graisse qui n'est pas bonne pour la fille... ils disent que lorsque tu fais le Depo-Provera avant d'avoir eu des enfants ce n'est pas bon pour toi. Ça peut manger tes... globules? (Marika). [M'pat vle mete'l. Paske yo di konsa presèvatif la gen yon Iwil ladan'l... ke gen yon grès ladan'l ki pa bon pou fi an... yo di konsa lè ou fè planing nan lè ou poko gen ti moun li pa bon pou ou. Li ka manje... globule ou, m'pa konnen.]

De telles croyances erronées permettraient en partie d'expliquer le faible taux d'utilisation de contraceptifs ainsi que le nombre élevé de grossesses précoces et non planifiées chez les femmes haïtiennes (Maynard-Tucker, 1996).

\section{Des grossesses précoces et non planifiées}

À la suite de l'annonce de leur grossesse, l'entourage de plusieurs participantes leur a conseillé de recourir à l'avortement clandestin. Les dangers associés à cette pratique artisanale, l'amour porté à l'enfant à naître (Legrand, 1990) et le sentiment de responsabilité en lien avec les croyances spirituelles (Jean-Charles, 2013) ont constitué des raisons de garder l'enfant, malgré les situations précarité vécues par ces femmes:

Les conseils que mes amis me donnaient... de boire un remède... pour le jeter... je disais non... s'il fallait qu'un problème grave arrive... je disais non, je ne vais pas le boire. Parce que je ne veux pas retirer l'enfant et j'aime l'enfant (Marika). [Konsèy zanmi'm tap ban mwen... pou'm bwè yon remèd... pou'm jete'l.... m'te di non... si yon pwoblèm grav ta rive... $m$ 'te di non, m'pap bwè'l. Paske m'pa vle retire ti moun nan e m'renmen ti moun nan.]

Je ne dois pas le retirer... je suis obligée de souffrir et d'endurer... parce que l'enfant ne t'a pas demandé de la faire... tu es obligée de répondre à toutes tes responsabilités. Comme le Bon Dieu me l'avait déjà donné et que je l'avais déjà pris, je ne pouvais pas m'en débarrasser... (Mirlyn). [Mwen pa dwe retire'l... m'oblije ret soufri pase misè... paske pitit la par mande'w pou ou fel... ou oblije repon'n a tout responsabilite'w. Kom Bon Dyé te gen tan ban mwen'l e ke m'te gen tan pran'l tou, m'pa ka jete'l m'oblije kimbe'l.]

Le nombre élevé de grossesses non planifiées menées à terme peut donc relever à la fois de l'influence des croyances religieuses, de la méconnaissance de la population en ce qui concerne la sexualité ainsi que de la valeur accordée à l'enfant. En effet, ce dernier représente une richesse, non seulement sur le plan émotionnel, mais aussi en ce qui concerne les perspectives d'avenir, celui-ci se devant d'assurer la subsistance de ses parents au cours de leur vieillesse (Maynard-Tucker, 1996).

\section{Des grossesses vécues dans l'isolement}

La grossesse précoce constitue un facteur d'isolement important chez ces jeunes Haïtiennes. En effet, elle représente une entrave à la poursuite de leurs études, en plus d'engendrer une rupture dans leurs relations amicales. Ces jeunes femmes sont délibérément retirées de l'école afin d'éviter de servir de mauvais exemple à leurs pairs (Laroche, 2012):

Avant que je ne tombe enceinte j'allais à l'école... j'avais quelques amis... on allait à l'école ensemble... et une fois que je suis tombée enceinte je ne suis plus allée à l'école (Mirlyn). [Anvan m'ansent $m^{\prime}$ te kon al lekol... m'te gen kek zanmi... nou te kon al lekol ansanm... kou m'tonbe ansent m'pa al lekol anko.] 
De plus, celles-ci vivent souvent du rejet de la part de leur famille, qui s'exprime par de la violence physique et psychologique ainsi que par des menaces d'abandon :

Elle me parlait très mal. Elle en est même venue à me dire de mourir avec l'enfant... elle me donnait des coups, me maltraitait... me disait qu'elle allait me mettre dehors... me parlait mal en public... (Mirlyn). [Li te pale'm trè mal. Li te menm arive di'm men pou ou mouri, ansanm ak pitit lan... li te kon bat mwen, li te maltrete'm... li te di'm ke l'tap mete'm deyo... li te pale'm mal devan moun.]

Toutefois, dans certains cas, les parents accepteront la grossesse, dans un second temps:

Quand elle a vu que mon ventre commençait à grossir elle a arrêté de m'insulter (Marika). [Lè li vin wè vant mwen komanse gwo li pa joure'm anko.]

En plus du risque d'être rejetées ou éloignées de leurs proches, plusieurs de ces jeunes mères se retrouvent en situation de monoparentalité, le père les ayant quittées dès l'annonce de la grossesse :

Je fréquentais un garçon... il est venu prendre ce qu'il voulait et quand je suis tombée enceinte il est parti (participante au groupe de discussion). [Mwen te renmen ak yon gason... sa li te bezwen li fin pran'l. Kounye a m'vin tonbe ansent l'ale.]

Dans d'autres cas, bien que le père de l'enfant soit physiquement présent et puisse même assurer le rôle de pourvoyeur, il tend néanmoins à fuir ou désinvestir son rôle parental :

Le père est là sans être là... il est là, mais c'est comme s'il n'était pas là (Marika). [Papa a la, se kom si'l pa la... li la men se tankou l'pat la.]

Par ailleurs, lors de nos observations participantes, nous avons pu voir le père de cet enfant entrer dans la maison pour ressortir aussitôt, avec une attention minimale accordée tant à la mère qu'à l'enfant.

Un tel désinvestissement de la part du père de l'enfant face à son foyer est chose commune en Haïti (FlambertChéry, 2013; Trouillot, 2013). En effet, il arrive à la majorité des hommes haïtiens de s'engager avec plusieurs partenaires à la fois, bien qu'ils le fassent de manière implicite, puisque la polygamie à proprement parler est interdite dans le pays et qu'elle y est décriée par le groupe religieux dominant (Lecarpentier et Letang, 2012).

Ne pouvant pas habiter avec chacune de leurs partenaires, ceux qui pratiquent cette polygamie déguisée ont généralement une conjointe ou une épouse avec laquelle ils demeurent officiellement et plusieurs autres compagnes à qui ils rendent visite occasionnellement (Joseph et Kahou, 2011). Le fait d'entretenir plusieurs unions à la fois (desquelles sont généralement issues plusieurs enfants) rend difficile pour ces hommes le plein investissement envers chacune d'entre elles (Flambert-Chéry, 2013; Trouillot, 2013).

De plus, bien que la Loi sur la paternité, la maternité et la filiation (MCFDF, 2014), visant à encourager la paternité responsable, ait été votée au Parlement haïtien en 2012, celle-ci n'est toujours pas entrée en vigueur et ne s'actualise donc pas dans les faits. Effectivement, d'une part, la possibilité d'intenter une procédure judiciaire afin de s'assurer que le père prenne ses responsabilités envers ses enfants est peu connue de la population haïtienne générale (notamment en raison d'une absence de campagnes de promotion et de sensibilisation par le gouvernement) (Bureau des Droits Humains en Haïti, 2016; Chancy, 2013) et, d'autre part, un tel recours se révèle très coûteux et donc peu accessible à la majorité des femmes haïtiennes (Joseph et Kahou, 2011).

Cela dit, bien que ces jeunes mères ne puissent pas toujours compter sur la présence effective du père de leur enfant, elles maintiennent néanmoins, la plupart du temps, des attentes financières à son égard. 


\section{Le père pourvoyeur : du père biologique au père substitut}

Compte tenu de la situation de grande précarité dans laquelle elles se trouvent, ces jeunes mères haïtiennes ont pour la majorité de la difficulté à répondre seules à leurs besoins ainsi qu'à ceux de leur enfant. Un sentiment de dépendance peut être vécu envers le père de leur progéniture, qui tient alors le rôle de pourvoyeur économique :

Quand il travaille, il me donne de l'argent pour l'enfant (Marika). [Lè li travay, li ban mwen pou piti la.]

Lorsque le père de l'enfant n'est pas à même de remplir ce rôle, certaines vont envisager le soutien financier d'un nouvel homme (Fitzgerald et collab., 2000; Maynard-Tucker, 1996; Smith-Fawzi et collab., 2005) :

Je ne dis pas que je ne le ferais jamais [me remettre en couple]... mais il faudrait qu'il s'agisse d'une situation extrême... si mon enfant ou moi venions à avoir un problème, je pourrais me mettre avec quelqu'un... mais à part ça... (Mirlyn) [M'pa di ke $m^{\prime}$ pap janm fèl non... men fo ta gen yon gwo ka... pou'm fèl. Si piti mwen genyon pwoblem, ou mwen menm tou, $m$ 'gen dwa ap renme... men aprè sa...]

Paradoxalement, cette option exposerait ces nouvelles mères à une éventuelle répétition de la maternité (Maynard-Tucker, 1996), de l'isolement et de leur situation de dépendance financière, facteurs risquant en fait d'augmenter leur situation de précarité.

Cette situation comporte également des risques pour l'enfant, en particulier les conséquences de la multiplicité des figures paternelles et de l'absence d'un milieu familial stable ainsi qu'une augmentation de la probabilité d'être placé en adoption par la mère ou confié à des personnes mieux nanties comme solution à la précarité (Lubin, 2002). Lorsque tel est le cas, l'enfant est le plus souvent condamné à la pauvreté et à l'exclusion (Joseph et Kahou, 2011), et parfois même réduit à des conditions d'esclavage (ces enfants sont appelés, en Haïti, des restavek) (Lubin, 2002).

Du reste, c'est à contrecœur que ces nouvelles mères se retrouvent dans de telles situations de dépendance :

J'aimerais que l'on fasse quelque chose pour moi. Pour moi et l'enfant... pour que je n'aie plus à demander aux gens... (Marika) [M'ta renmen pou nou ta fè yon bagay pou mwen. Pou mwen menm ansanm avek ti moun nan. Pou'm pa nan mande moun.]

\section{Un soutien psychique limité}

En parallèle aux ruptures et au soutien limité par les proches, ces jeunes mères haïtiennes semblent puiser force et réconfort au sein de leurs valeurs et de leurs croyances :

Lorsque tu souffres, il peut y avoir un problème qui te tracasse... mais n'oublie pas que tout le temps que tu vis il y a de l'espoir... (Mirlyn). [Loske wap soufri ou ka gen yon pwoblem kap trakase'w... men pa bliye tou tan wap viv gen espwa.]

Au niveau psychique, la figure de Dieu peut représenter pour ces femmes une présence positive pouvant combler un manque relationnel :

II y a plein de dangers au cours d'un accouchement... s'il m'arrivait de mourir... je priais Dieu beaucoup... pour que ce genre de choses ne m'arrivent pas (Mirlyn). [Gen anpil danje nan akouchman... si $\mathrm{m}^{\prime}$ te arive mouri... m'te kon prie Bon Dye anpil... pou bagay sa yo pat rive'm.]

Ainsi, bien que les croyances et l'influence de l'Église aient pu avoir un rôle à jouer dans la survenue des grossesses non planifiées et souvent non désirées chez nos participantes, celles-ci semblent, après coup, avoir un impact positif (rarement mentionné dans la littérature) sur la résilience de ces dernières quant aux difficultés associées à leur grossesse. II semble toutefois que le soutien spirituel sur lequel ces mères s'appuient ne puisse totalement compenser les sentiments dépressifs qui les habitent. 


\section{De la grossesse à la maternité : prégnance des affects dépressifs}

Les participantes nous ont confié avoir fait l'expérience de pensées négatives durant leur grossesse en raison des circonstances ayant entouré cette dernière, en particulier en ce qui a trait aux conflits familiaux (teintés de violence) :

Je pensais à plein de choses et parfois... je me disais que j'avais envie de me suicider... (Mirlyn) [M'te kon ap pense anpil e pafwa... m'te kon ap di'm, m'anvi tuye tèt mwen...]

De plus, les considérations précédentes sur les aléas de leur maternité - soit le manque de soutien et de ressources pour élever un enfant, l'isolement, la dévalorisation ou la désapprobation familiale ainsi que la monoparentalité ou le désinvestissement du père de l'enfant - amènent plusieurs jeunes mères à poser un regard négatif sur leur expérience de la maternité. En ce sens, la grossesse est évoquée par Mirlyn avec une connotation particulièrement négative :

Comme les adultes disent, un malheur ne nous prévient pas avant d'arriver. Cette erreur ne pourra jamais être réparée. [Kom gran moun yo di, lè malè a rive li pa gen klaksone. Ere sa pap janm ka repare.]

En outre, le patriarcat et les mentalités machistes étant toujours fortement ancrés dans la culture haïtienne (Edouard, 2013; Prédestin, 2006; Solidarite fanm hayisyèn, 2011), ces femmes disposent de peu de support dans leur rôle de mère :

Moi, mes parents ne m'ont donné aucune information sur les enfants. Faire l'éducation c'est lourd... une mère doit veiller à tout. Tout le travail revient à la mère. Une mère de famille est toujours active. Parce que le père lui n'est pas toujours là. La mère est obligée de rester là comme une esclave (participante au groupe de discussion).

De ce fait, l'enfant représente, pour plusieurs de ces mères, une augmentation de la souffrance et des difficultés, en plus de constituer un obstacle à la réalisation de leur rêve de terminer leurs études. Cela rejoint d'ailleurs les idéaux familiaux : la valeur accordée à l'éducation en tant que voie d'accès vers un avenir meilleur expliquant l'ampleur de la réaction (violence, rejet) à l'annonce de la grossesse décrite précédemment (Cénat, Derivois et Merisier, 2010).

Le vécu dépressif demeure toutefois privé (de même que, parfois, l'ampleur de la précarité) afin d'éviter de revivre du rejet de la part de leur famille et par peur de l'indiscrétion du voisinage propre aux petites communautés :

Il y a des fois où je n'avais rien et où je restais là à souffrir... mais je ne le disais pas, c'était personnel à moi. Parce que je sais qu'une fois que je l'aurais dit, ils auraient été l'ébruiter dehors... je ne voulais pas en parler à qui que ce soit, pas même à mes propres gens. Parce que... si je leur avais dit, ils m'auraient insultée (Mirlyn). [Gen de fwa m'pat kon jwèn tou, m'rete la m'soufri. M'pat kon esplike moun anyen, sa te pesonel a mwen. M'pat vle pale de sa a peson. Menm a moun pam m'pat vle di'l. Paske si map di'l yap jouré'm.]

Bien que ce ne soit pas le cas chez la totalité nos participantes, le vécu dépressif maternel a persisté chez certaines suite à la naissance de l'enfant, ce qui a pu avoir un impact négatif significatif sur le développement de l'enfant et en particulier sur le lien d'attachement mère-enfant, qui joue un rôle capital dans le développement psychique (Guyotat, 2005).

\section{Maternité en situation de précarité et liens d'attachement}

Malgré le contexte global de précarité et d'absence de planification entourant l'expérience de la maternité chez ces jeunes mères, des liens d'attachement fort différents ont été observés d'une dyade mère-enfant à une autre. Nous avons pu constater, à travers nos groupes de discussion ainsi que par l'observation à domicile, que certaines mères, bien que leur grossesse ne fût pas planifiée, témoignaient d'un important investissement envers leur enfant.

Chez Marika en particulier, nous avons relevé une réponse adéquate aux besoins de son enfant (sur les plans affectifs, de la nourriture, de la communication et de l'intérêt au jeu) en plus de la présence d'un sentiment de 
fierté par rapport à son nouveau rôle. Paradoxalement, cet investissement affectif évident envers un enfant représentant une richesse pour la mère était associé à un certain désinvestissement et à des signes de négligence au niveau des soins physiques. En effet, ce dernier était couvert de poussière et jouait sur le sol au milieu de fourmis, non loin d'un monticule de déchets, ce qui pourrait entre autres s'expliquer par une méconnaissance par la mère de certains soins et précautions nécessaires.

À l'inverse, selon d'autres participantes à nos groupes et par l'observation à domicile effectuée chez Mirlyn, il semble que plusieurs mères vivent un certain désespoir en lien avec les difficultés inhérentes à leur situation de précarité, ce qui influence le lien affectif à leur enfant. Ce lien, dès lors teinté d'ambivalence, les pousserait, comme cela a été mentionné ci-dessus, à envisager de placer leur enfant en adoption ou à le confier à des gens qui risquent de l'utiliser comme restavek:

On dirait que je l'aime beaucoup... je la trouve très intéressante. Je l'aime beaucoup. Mais parfois... il m'arrive de me dire que j'ai envie de la donner (Mirlyn). [Gen lè m’renmen'l anpil... m’trouve'l tre interesant. M'renme'l anpil. Men pafwa... m'kon di'm ke m'anvi ba li.]

Chez Mirlyn l'ambivalence envers son enfant paraît refléter le difficile investissement du rôle d'adulte, de mère. Adopter une posture d'enfant en quête de prise en charge semble cohérent avec la dépendance socioéconomique de ces jeunes mères envers leurs parents: en demeurant dans la place d'enfants de ces derniers, comme ils peuvent le percevoir, ces jeunes mères s'identifient à leur progéniture plutôt que d'accepter la permutation des places sur le plan générationnel, ce qui suscite chez elles une posture régressive :

Si une personne prend ma fille, elle va me prendre en même temps! (Mirlyn) [Si yon moun pran piti mwen lap tou pran'm tou!]

Sur le plan psychique, nous avons noté chez Mirlyn un profond désinvestissement par rapport à sa progéniture. En effet, cette nouvelle mère démontrait une attitude détachée en présence de son enfant, ainsi qu'une absence de réponse à certains besoins d'ordres affectif, nutritionnel et de sécurité. Le regard absent, cette mère tenait sa fille de façon instable, tout en ignorant ses pleurs. Quant à l'enfant, elle avait elle aussi le regard vide et ne semblait pas réagir aux interactions.

Toutefois, Mirlyn dévoilait par moments une représentation positive de sa maternité. Par exemple, elle a mentionné que son enfant suscitait sa curiosité, qu'elle constituait pour elle une motivation à rester en vie et qu'il lui arrivait de ressentir une forme d'apaisement lorsqu'elle se trouvait en sa présence, en plus d'éprouver un sentiment de fierté par rapport à son rôle de mère :

Je dis toujours au Bon Dieu : "Ne me laisse pas mourir maintenant parce que je ne veux pas quitter l'enfant ». Parce qu'avec tout ce que j'ai subi... je n'ai pas envie qu'elle vive cela. S'il m'arrivait de mourir... je viendrais la chercher aussi. [M'toujou di Bon Dye : pa kite'm mouri kounya pou $\mathrm{m}^{\prime}$ pa kite pitit la. Paske pou misè $\mathrm{m}^{\prime}$ pase... $\mathrm{m}$ 'pa ta renmen pou'l pase misè kon sa. Si $\mathrm{m}^{\prime}$ te rive mouri $\mathrm{m}^{\prime}$ tap vin cheche'l.]

\section{Discussion}

Notre recherche permet d'accéder à une compréhension plus juste des histoires de vie ainsi que des difficultés auxquelles sont confrontées les jeunes mères utilisatrices des services offerts par GROSAME. Ce faisant, on peut désormais envisager une meilleure adaptation de l'intervention à leur réalité complexe et à leurs besoins.

L'intervention pourrait inclure un volet préventif qui tienne compte des facteurs de risques socioculturels (dont le patriarcat, les tabous culturels et spirituels, l'éducation sexuelle limitée ainsi que la faible utilisation de contraceptifs et la difficulté d'accès à l'avortement) des grossesses accidentelles en Haïti. En outre, une attention particulière devrait être portée au vécu de ces jeunes femmes autour de leur maternité, tant au niveau social (marqué par l'isolement, l'absence de soutien, le rejet et la dépendance) qu'au niveau individuel (notamment en ce qui concerne le lien mère-enfant). 


\section{Les facteurs de risques socioculturels}

Les tabous entourant la sexualité en Haïti donnent lieu à une méconnaissance entourant les méthodes contraceptives chez plusieurs jeunes femmes haïtiennes. Cette méconnaissance, jumelée à leurs attentes et à leur dépendance envers leur partenaire, réduit le contrôle de ces dernières sur leur sexualité, tout en exacerbant le risque pour elles de vivre une grossesse précoce et non planifiée.

Un moyen efficace de contrer cette tendance, en tenant compte notamment du fort taux d'analphabétisme en Haïti (Guay, 2016), demeure la promotion de l'éducation sexuelle par la radio. Un pas en ce sens a été posé par GROSAME durant les deux dernières années, avec la création d'émissions hebdomadaires abordant notamment la sexualité et la grossesse (Gilbert et collab., 2015).

Cela dit, cette initiative se heurte à de nombreux obstacles historiques, politiques et économiques, tels que le patriarcat et le peu de soutien (notamment monétaire) accordé par l'État à la prévention. À court terme, il demeure donc difficile de prévenir, autrement que par des initiatives locales - de ce fait limitées - les grossesses précoces en situation de grande précarité ainsi que les difficultés qui y sont associées.

\section{Le vécu entourant la maternité}

Rappelons que lorsque survient une grossesse non planifiée, les jeunes femmes qui choisissent de mener celle-ci à terme vivent régulièrement des situations de ruptures multiples. En découlent une solitude et une souffrance importantes chez ces dernières, susceptibles d'avoir d'importantes répercussions sur le lien mère-enfant et sur le développement ultérieur de ce dernier.

En effet, un élément fondamental de nos résultats est la mise en lumière de la prévalence de l'ambivalence des mères face à la maternité, surtout face à leur enfant, sur qui elles projettent le plus souvent cette ambivalence (Pines, 1982). D'une part, cette ambivalence porte sur le choix conscient de mener ou non la grossesse à terme - ici s'opposent notamment les risques associés à l'avortement clandestin et le désir de se sortir de la misère, en gardant un homme près de soi. D'autre part, l'ambivalence du désir d'enfant (de nature inconscient) nourrit des sentiments opposés face à l'enfant (Bydlowski, 2008).

En matière d'enjeux intrapsychiques (échappant à la conscience), l'ambivalence des nouvelles mères pourrait s'avérer sous-tendue par l'inscription de la grossesse dans une "filiation narcissique, elle-même régie par une logique de la reproduction à l'identique » (Guyotat, 2005). Certaines de ces mères seraient donc exposées, malgré leur volonté, et par leur enfant, à la répétition de leur propre histoire.

En ce sens, nous avons relevé combien leur expérience de la maternité semble reproduire l’absence de leur propre père et, ainsi, constituer une possible actualisation des conflits de leur enfance, malgré l'espoir d'y remédier. Si le père de l'enfant apparaît d'abord à ces mères comme une solution à leur situation de précarité actuelle, et offre un espoir de se différencier de leur propre vécu infantile, elles reproduisent, parfois à plusieurs reprises et sans le vouloir, la précarité et la monoparentalité de leur enfance (Gilbert et collab., 2015). En découlent des sentiments mitigés envers l'enfant à la source de ce vécu empreint de souffrance.

À l'inverse, ce même désir de se différencier des figures parentales, notamment au travers de l'enfant, pourra alimenter une posture protectrice envers ce dernier. Ainsi, l'une des mères rencontrées a expliqué que, bien qu'elle ait pensé parfois donner son enfant en adoption, la remémoration de son propre vécu de maltraitance chez des parents de substitution dans l'enfance l'en a empêché. Elle craignait qu'en renonçant à cette enfant (à laquelle elle semblait s'identifier), cette dernière se retrouve aux prises avec des figures parentales maltraitantes, reproduisant ainsi sa propre expérience.

De plus, imaginer une éventuelle séparation d'avec son enfant semblait réactiver chez elle la souffrance associée aux nombreuses ruptures et situations de rejet ayant ponctué son histoire. On constate donc chez cette mère le désir (également relevé chez des mères occidentales en situation de précarité) de ne pas répéter avec son enfant 
une histoire empreinte de vécus difficiles, voire traumatiques (Gilbert et collab., 2015) - qui dans ce cas, motive un investissement plus important envers l'enfant.

Par ailleurs, la grossesse peut représenter une occasion pour ces nouvelles mères de restaurer leur narcissisme (Bruwier, 2012). En effet, elles peuvent entretenir un sentiment de bien-être et de fierté par rapport à leur enfant et au fait de devenir mère, d'autant plus qu'en Haïti, la valeur positive accordée à l'enfant fait en sorte que la fertilité représente un atout important chez les femmes (Maynard-Tucker, 1996).

Ces affects positifs - fierté relative à la grossesse ou, potentiellement, au rôle de mère protectrice - constitueraient une voie à saisir dans l'intervention. Toutefois, ils ont coexisté chez nos participantes avec des affects nettement dépressifs, en raison de l'isolement et du peu d'attention accordée à la dyade mère-enfant par le père de ce dernier, mais aussi, et surtout, en raison de la dévalorisation de leur expérience de la maternité régnant au sein de leur cellule familiale et s'exprimant, rappelons-le, par du rejet, un refus d'acceptation, de la violence psychologique et physique ou encore des menaces d'abandon.

Entrant fortement en contradiction avec la valorisation culturelle généralement attribuée au rôle de mère en Haïti, cette dévaluation de l'expérience de la maternité s'explique, comme nous l'avons mentionné précédemment, par la nature précoce des grossesses de nos participantes, qui constitue une entrave à la poursuite des études, particulièrement valorisées par les parents comme voie d'accès à un avenir meilleur (Cénat, Derivois et Merisier, 2013).

Ainsi, l'ambivalence de nos participantes pourrait s'avérer renforcée par l'absence de soutien entourant leur maternité. En effet, la venue d'un enfant demande une disponibilité ainsi qu'un investissement important de la donneuse de soins pouvant l'amener à se sentir dépassée et conséquemment, entacher sa relation avec lui. Selon Bruwier (2012, p.41), « de par sa dépendance, le bébé exige une disponibilité physique et psychique telle qu'une mère peut se sentir complètement accaparée par cet "amour impitoyable" (Winnicott, 1969) et ressentir de l'agressivité pour ce bébé "qui pompe tout" (Winnicott, 1969) ».

En fait, même les mères ayant vivement désiré un enfant sont susceptibles de vivre de l'ambivalence envers celuici. L'une des participantes a d'ailleurs affirmé se sentir étourdie et " desséchée » à mesure que l'enfant tétait. Une image qui dépeint bien la complexité de l'allaitement maternel au sein des populations défavorisées et, de ce fait, malnutries, et ses conséquences sur la santé psychique et physique de la mère, lorsqu'il est pratiqué sans encadrement et sans l'administration de suppléments alimentaires (Organisation des Nations unies pour l'éducation, la science et la culture, 1984).

Dans le même ordre d'idées, une autre participante s'autoqualifiait de "mère esclave ". Une telle image de mère condamnée à tout faire pour l'enfant semble ici paradoxale, compte tenu du fait que certaines de ces mères ont démontré un désir d'être d'abord elles-mêmes prises en charge par une "figure parentale ", que ce soit leur conjoint (présent ou futur), GROSAME ou la chercheure. La difficulté pour ces femmes de renoncer à la position d'enfant qu'elles occupaient avant l'arrivée de leur progéniture, afin d'assumer leur posture de mère à part entière, pourrait être à relier au fait qu'elles n'ont pas pu bénéficier d'une transition entre ces deux rôles.

En effet, en raison de leur jeune âge, de leur situation de dépendance financière ou encore d'une quasi-absence de modèle en ce sens, il aurait été difficile pour elles d'apprendre d'abord à s'occuper d'elles-mêmes en tant que femmes émancipées, ce qui complexifierait la tâche de s'occuper de quelqu'un d'autre, en l'occurrence de leur enfant. En Haïti, cette absence d'espace permettant d'être femme, et non pas seulement fille ou mère, dévoile le rôle social auquel sont le plus souvent cantonnées les femmes, soit celui de reproductrices et, par extension, de ménagères et d'épouses (MCFDF, 2006).

Ainsi, une manière pour GROSAME de répondre aux besoins des utilisatrices (outre les services déjà en place) serait de soutenir l'investissement affectif envers l'enfant en tenant compte des affects dépressifs relevés chez les mères, qui teintent l'investissement de leur rôle maternel. De plus, il s'agirait pour les intervenantes de leur proposer non seulement un modèle maternel indirect (par la formation) mais également un modèle affectif réparateur 
(présence, affection) qui pourrait être transmis à la progéniture dans une perspective intergénérationnelle. Plus largement, il s'agirait d'agir au niveau familial et socioculturel d'une part en impliquant la famille des jeunes femmes dans l'intervention et, d'autre part, en soutenant les motivations culturelles et spirituelles à la maternité déjà présentes chez celles-ci, comme levier à la résilience.

\section{Conclusion}

Malgré le caractère exploratoire de cette étude, nos résultats permettent de faire ressortir certains points saillants de la trajectoire des nouvelles mères utilisatrices des services offerts par GROSAME, leurs difficultés, de même que certains de leurs besoins psychosociaux en lien avec ceux de leur enfant. Nous avons pu à partir de là envisager des pistes d'interventions en vue d'améliorer les services offerts à cette population et, dans une visée plus large, d'alimenter une réflexion critique et éclairée sur la situation des jeunes mères haïtiennes en situation de très grande vulnérabilité socioéconomique, dans un contexte où peu d'études ont été menées sur le sujet.

En outre, notre recherche met en évidence que l'absence ou le désinvestissement psychique des pères entourant la maternité (ayant été relevé de manière généralisée au sein du discours de nos participantes) mériterait d'être exploré davantage. En effet, le pouvoir des femmes passe aussi par une prise en compte de la réalité des hommes ainsi que par une éventuelle modulation de leur conception du rôle de père. D'autre part, il nous apparaît clair, à l'issue de cette recherche, que des données plus importantes seraient nécessaires à une compréhension plus extensive de la situation des nouvelles mères haïtiennes en 2017, mais aussi à l'élaboration de services qui pourraient leur redonner du pouvoir sur leur vie de mère et de femme.

Pour terminer, rappelons que la principale force de notre étude consiste en la qualité et la profondeur de nos données, tributaires de l'attention particulière portée aux éléments culturels ayant pu teinter aussi bien le discours de nos participantes que l'interprétation que nous en avons faite par la suite. En effet, nous avons traduit les entretiens du créole au français en nous efforçant d'en garder l'essence et la signification, et en prenant soin de conserver certains détails tels que les hésitations. De plus, les discussions informelles que nous avons eues avec les intervenants de l'organisme nous ont permis de comprendre certains aspects de la réalité des utilisatrices, du milieu d'intervention et, plus largement, de la culture et des valeurs propres à la communauté haïtienne.

\section{Références bibliographiques}

Benarous, X, Guilé J.-M., Consoli, A. et Cohen, D. (2014). A systematic review of the evidence for impaired cognitive theoryof mind in maltreated children. Front Psychiatry, 108(6). https://www.ncbi.nlm.nih.gov/pmc/articles/PMC4516890/

Bruwier, G. (2012). La grossesse psychique : I'aube des liens. Bruxelles : Fabert. http://www.yapaka.be/sites/yapaka.be/files/55_grossesse-web.pdf

Bureau des Droits Humains en Haïti. (2016). Le cheminement de la réforme sur la paternité responsable. http://bdhhaiti.org/le-cheminement-de-la-reforme-sur-la-paternite-responsable/

Bydlowski, M. (2008). Les enfants du désir : le désir d'enfant dans sa relation à l'inconscient. Paris: Odile Jacob.

Cénat, J. M., Derivois, D. et Merisier, G. G. (2013). École et résilience chez les adolescents dans l'Haïti postséisme. Revue québécoise de psychologie, 34(2), 189-201.

https://www.researchgate.net/publication/262877209_ECOLE_ET_RESILIENCE_CHEZ_LES_ENFANTS_ET_AD OLESCENTS_DANS_L'HAITI_POSTSEISME

Chancy, A. (2013). La part des hommes. Dans Conjuguer les genres pour lutter ensemble contre les violences faites aux femmes. Programme des Nations Unies pour le développement (PNUD). http://www.ht.undp.org/content/dam/haiti/docs/emancipation_des_femmes/Guide\%20Masculinite.pdf

Chandra-Mouli, V., Camacho, A. V. et Michaud, P. A. (2013). WHO Guidelines on Preventing Early Pregnancy and Poor Reproductive Outcomes Among Adolescents in Developing Countries. Journal of adolescent health, 52(5), 517-522. http://www.jahonline.org/article/S1054-139X(13)00121-3/fulltext 
Edouard, R. (2013). Violence et ordre social en Haïti: essai sur le vivre ensemble dans une société postcoloniale. Québec : Presses de I'Université du Québec.

Fitzgerald, D.W. et al. (2000). Economic hardship and sexually transmitted diseases in Haiti's rural Artibonite valley. The American journal of tropical medicine and hygiene, 62(4), 496-501. http://www.ajtmh.org/content/journals/10.4269/ajtmh.2000.62.496

Flambert-Chéry, M. C. (2013). Le père absent: études psychologiques sur la famille haïtienne. Port-au-Prince : Université d'État en Haïti.

Gage, A. J. et Hutchinson, P. L. (2006). Power, control, and intimate partner sexual violence in Haiti. Archives of Sexual Behavior,35 (1), 11-24. https://www.ncbi.nlm.nih.gov/pubmed/16502150

Gilbert, S., Benjamin, F., Da, J. L., Toussaint, J. M. et Lecomte, Y. (2015). Perspectives sur la résilience... collective : créer un réseau communautaire en santé mentale à Grand-Goâve, Haïti. Revue haïtienne de santé mentale, (4), 85-106. http://grosamegrandgoave.com/wp-content/uploads/2016/01/6.-Gilbert-et-al.pdf

Gilbert, S. (2009). La recherche qualitative d'orientation psychanalytique : I'apport heuristique des rencontres intersubjectives. Recherches qualitatives, 28(3), 19-39. http://www.recherchequalitative.qc.ca/documents/files/revue/edition_reguliere/numero28(3)/Sophie_Gilbert28(3).pdf

Grosame Grand-Goâve. (2016). Nouvelles familles. http://grosamegrandgoave.com/2016/02/03/nouvellesfamilles/http://grosamegrandgoave.com/2016/02/03/nouvelles-familles/

Guay, J. H. (2016). Degré d'alphabétisation - adultes (\% des adultes âgés de plus de 15 ans), Haïti. Perspective monde. Sherbrooke : Université de Sherbrooke.

Guyotat, J. (2005). Traumatisme et lien de filiation. Dialogue, 166(2), 15-24. http://www.cairn.info/revue-dialogue2005-2-page-15.htm

Jean-Charles, V. (2013). Profils identitaires et prises de position sur la sexualité d'adolescent(e)s pentecôtistes et catholiques pratiquant(e)s de Port-au-Prince (Haïti) : analyse d'un champ représentationnel en fonction des modes d'insertion sociale. (Thèse de doctorat, Université Laval à Québec, Canada). file://C:/Users/Asus/Downloads/29581\%20(2).pdf

Joseph, K. et Kahou, P. F. (2011). Haïti - État de la situation des femmes : pré et post séisme 2010. http://sisyphe.org/spip.php?article3804

Laroche, A. (2012). Haïti-Société-Sante : La grossesse précoce une catastrophe sociale. Haïti Press Network. http://www.hpnhaiti.com/site/index.php/societe/5962-haiti-societe-sante-la-grossesse-precoce-unecatastrophe-sociale

Lecarpentier, P. et Letang, J. (2012). La " paternité responsable » : une réforme essentielle mais une loi problématique? Le Nouvelliste. http://lenouvelliste.com/lenouvelliste/article/111559/La-paterniteresponsable-une-reforme-essentielle-mais-une-loi-problematique

Legrand, B. (1990). Coup d'œil sur la famille haïtienne. Port-au-Prince : Éditions des Antilles.

Logeart, A. (2014). "Tomber la grossesse" : I'enfer des avortements clandestins à Haïti. L'OBS Monde. http://tempsreel.nouvelobs.com/monde/20140919.OBS9753/tomber-la-grossesse-l-enfer-des-avortementsclandestins-a-haiti.html

Lubin, I. (2002). Un regard sur la domesticité juvénile en Haïti. Refuge, 20(2), 45-51. http://refuge.journals.yorku.ca/index.php/refuge/article/view/21253

Maternowska, M.-C. (2006). Reproducing inequities : Poverty and the politics of population in Haiti. New Brunswick (NJ) : Rutgers University Press.

Maynard-Tucker, G. (1996). Haiti: Unions, fertility and the quest for survival. Social Science and Medicine, 43(9), 1379-1387. https://www.ncbi.nIm.nih.gov/pubmed/8913007 
Ministère à la Condition Féminine et aux Droits des Femmes. (2006). Les termes de la problématique de Genre en HAÏTI. Eléments de la Condition et des Situations des Femmes en Haïti.

http://www.haitivisions.com/mcfdf/condition_feminine.pdf

Ministère à la Condition Féminine et aux Droits des Femmes. (2014). Loi sur la paternité, la maternité, et la filiation. https://www.healthpolicyproject.com/pubs/713_BrochurePaternitewithcover.pdf

Ministère de la Santé Publique et de la Population (2013). L'Enquête Mortalité, Morbidité et Utilisation des Services en Haïti de 2012 : Rapport de synthèse. Pétion Ville, Haïti et Maryland, États-Unis : MSPP, IHE et ICF International. http://haiti.unfpa.org/sites/default/files/pub-pdf/JMP2013_Rapport_de_synthEse_Haiti.pdf

Organisation des Nations unies pour l'éducation, la science et la culture (Unesco). (1984). La nutrition de la mère et du petit enfant. Paris : Unesco. http://unesdoc.unesco.org/images/0005/000545/054551fo.pdf

Organisation mondiale de la Santé. (2014). La maltraitance des enfants. Centre des médias. http://www.who.int/mediacentre/factsheets/fs150/fr/.

Paillé, P. et Mucchielli, A. (2012). L'analyse qualitative en sciences humaines et sociales. Paris : Armand Colin.

Paul, B., Dameus, A. et Garrabe, M. (2011). Le processus de tertiarisation de l'économie haïtienne. Études caribéennes, 16. http://etudescaribeennes.revues.org/4757

Prédestin, J. M.-C. (2006). La situation de la femme en Haïti au regard des instruments nationaux et internationaux [Internet]. Mémoire online. http://www.memoireonline.com/02/08/955/m_situation-femme-haitiinstruments-nationaux-internationaux3.html

Pines, D. (1982). The relevance of early psychic development to pregnancy and abortion. Amercian psychological association, 63(3), 311-319. https://www.ncbi.nlm.nih.gov/pubmed/7129777

Richard, P. J. (2015). Haïti : la marche à suivre pour la maternité sans risque encore longue. MINUSTAH. https://minustah.unmissions.org/ha\%C3\%AFti-la-marche-vers-la-maternit\%C3\%A9-sans-risque-encorelongue

Smith-Fawzi, M. C. et al. (2005). Factors associated with forced sex among women accessing health services in rural Haiti. Social Science and Medicine, 60(4), 679-689.

http://www.sciencedirect.com/science/article/pii/S0277953604002795

Solidarite fanm hayisyèn. (2011). Des cas de violence accueillis et accompagnés dans les centres d'accueil de la SOFA. (Rapport, Bilan XI). http://www.alainet.org/images/SOFA\%20-Onzieme\%20Rapport\%20Bilan\%20\%20decembre\%202011-2.pdf

Trouillot, É. (2013). Rôle de la mère dans la transmission des valeurs et la construction de la masculinité. Dans Conjuguer les genres pour lutter ensemble contre les violences faites aux femmes (p. 51-58). Programme des Nations Unies pour le développement (PNUD). http://www.ht.undp.org/content/dam/haiti/docs/emancipation_des_femmes/Guide\%20Masculinite.pdf

Winnicott, D. W. (1950-1955). L'agressivité et ses rapports avec le développement affectif. Dans : De la pédiatrie à la psychanalyse. Paris : Payot.

Winnicott, D. W. (1969). La haine dans le contre-transfert. Paris : Payot/Rivages. 